\title{
Global solution to Cauchy problem of fractional drift diffusion system with power-law nonlinearity
}

\author{
Caihong $\mathrm{Gu}^{1}$ and Yanbin Tang ${ }^{1}$ \\ ${ }^{1}$ Huazhong University of Science and Technology
}

June 23, 2021

\begin{abstract}
In this paper we consider the global existence, regularizing-decay rate and asymptotic behavior of mild solutions to the Cauchy problem of fractional drift diffusion system with power-law nonlinearity. Using the properties of fractional heat semigroup and the classical estimates of fractional heat kernel, we first prove the global-in-time existence and uniqueness of the mild solutions in the frame of mixed time-space Besov space with multi-linear continuous mappings. Then we show the asymptotic behavior and regularizing-decay rate estimates of the solution to equations with power-law nonlinearity by the method of multi-linear operator and the classical Hardy-Littlewood-Sobolev inequality.
\end{abstract}

\section{Hosted file}

GuCaiHong20210606.pdf available at https://authorea.com/users/421397/articles/527454global-solution-to-cauchy-problem-of-fractional-drift-diffusion-system-with-power-lawnonlinearity 